\title{
Periodic orbits and warps
}

\author{
W. A. Mulder
}

Sterrewacht, Huygens Laboratorium, P.O. Box 9513, NL-2300 RA Leiden, The Netherlands

Received October 15, 1982; accepted January 18, 1983

Summary. Orbit calculations have been carried out in a rotating triaxial system having a density distribution in accordance with recent observations of spiral galaxies. A search was made for simple closed orbits that are tilted with respect to the equatorial plane of the galaxy. A new family of stable prograde tilted orbits was found which can explain warps as almost stationary phenomena.

The results give strong support to Binney's proposal that warps can be excited by orbital instability. A scenario is described in which gas in the equatorial plane slowly spirals inward, is fed onto the prograde tilted orbits by orbital instability and eventually ends up as high-velocity clouds due to the intersection of the more inclined orbits.

No contradictions were found when the characteristic properties of the orbits were compared with the warps of our Galaxy and NGC 2903.

Key words: triaxial systems - periodic orbits - warps - NGC 2903 - tilted rings

\section{Introduction}

Tilted disks are a common phenomenon observed in many early type and elliptical galaxies (Bertola et al., 1978; Hawarden et al., 1981). A tilted distribution of gas is also seen near the galactic centre (Liszt and Burton, 1980). Spiral galaxies often show gaseous warps (e.g. Bosma, 1978). Attempts have been made to explain these configurations in terms of stable closed orbits in rotating triaxial systems. Merritt (Heisler et al., 1982) found a family of retrograde tilted orbits in a slowly rotating system. A model for the dust lanes in Centaurus A, based on these orbits, was proposed by Van Albada et al. (1982). Binney $(1978,1981)$ used Mathieu's equation to show that orbits in the plane of a rotating triaxial potential can be unstable for perturbations perpendicular to that plane.

In this paper, the most important results of a search for simple stable tilted orbits in a rotating triaxial system are reported. The density distribution was chosen in accordance with recent observations of spiral galaxies and of the bulge of our Galaxy. Emphasis was placed on simple orbits because those are most likely to be populated by gas. This excludes orbits that are selfintersecting, have sharp turnings or a complex shape, or are asymmetric with respect to the origin. A new important family of prograde tilted orbits was found, which can be used to explain warps as almost stationary phenomena.
The potential that was used is given in Sect. 2. Orbits in this potential were calculated with the method given by Magnenat (1982b).

The resulting orbits are given in Sect. 3. The importance of the family of orbits found is investigated by applying results to the warps of our Galaxy and NGC 2903 in Sect. 4. Section 5 contains the main conclusions.

\section{Method}

The triaxial density distribution is chosen to be:

$\varrho=\varrho_{0} m^{p}$, with $m^{2}=x^{2} / a^{2}+y^{2} / b^{2}+z^{2} / c^{2}$ and $a>b>c$.

Near-infrared observations of the galactic bulge by Becklin and Neugebauer (1968) were used by Sanders and Lowinger (1972) to derive values of $p=-1.8, \varrho_{0}=7.610^{5} M_{\odot} \mathrm{pc}^{-3}$ at $1 \mathrm{pc}$ and an axial ratio $c / a=0.4$ within $800 \mathrm{pc}$. Using the distribution of planetary nebulae near the galactic centre, Isaacman (1981) found: $p=-1.8 \pm 0.3, c / a=0.4$, and $M=(9 \pm 2) 10^{9} M_{\odot}$ within $1 \mathrm{kpc}$. Finally, Burstein et al. (1982) found $p=-1.7 \pm 0.1$ from the rotation curves of a sample of $21 \mathrm{Sc}$ galaxies. The density distribution appears to keep this value even at large radii. Therefore, bulge and halo are modelled here with a single density distribution. The disk mass is neglected since this seems justified by the analysis of neutral hydrogen in the outer Milky Way by Kulkarni et al. (1982). They find a large increase in scale height with radius, suggesting that the large mass of non luminous matter implied by their rotation curve must reside outside the disk. A similar conclusion with different arguments is reached by Van der Kruit and Searle (1982) on the basis of photometry of edge on spirals.

The following parameters are chosen: $p=-1.8$, an axial ratio $c / a=0.5$ for the minor axis and $b / a=0.8$ for the intermediate. The triaxial figure is oblate with a moderate oval distortion. It rotates around the $z$-axis (the minor axis) with a rotation frequency $\omega=0.1$, implying co-rotation $(\mathrm{CR})$ to be at a radius $r_{\mathrm{CR}}=8.37$.

The triaxial density distribution results in a potential (using theorem 12 from Chandrasekhar, 1969):

$V(r, \theta, \varphi)=C r^{p+2} I(\theta, \varphi)$,

with

$C=4 \pi G \varrho_{0} \frac{b c}{(p+2) a^{p+2} \kappa^{p+3}}$

and

$I(\theta, \varphi)=\int_{0}^{\kappa} d x \frac{x^{p+2}\left[1+v x^{2}+\mu x^{4}\right]^{(p+2) / 2}}{\left[\left(1-x^{2}\right)\left(1-\lambda x^{2}\right)\right]^{(p+3) / 2}}$ 
Table 1. Approximate locations and energies of the main resonances

\begin{tabular}{llccccc}
\hline$\omega$ & $l / k$ & $r_{k: l: *}$ & $E_{k: l: *}$ & $m / k$ & $r_{k: *: m}$ & $E_{k: *: m}$ \\
\hline-0.1 (retro) & $1 / 2$ & 17.7 & 6.08 & $1 / 2$ & 12.1 & 5.19 \\
& 1 & 3.73 & 3.56 & 1 & 1.35 & 2.77 \\
0.1 (progr) & 2 (ILR) & 1.86 & 2.72 & 2 & 3.05 & 2.92 \\
& 3 & 3.92 & 3.00 & 3 & 4.76 & 3.06 \\
& 4 & 5.00 & 3.08 & 4 & 5.65 & 3.10 \\
& $\infty(\mathrm{CR})$ & 8.37 & 3.15 & & & \\
& -4 & 11.9 & 3.10 & -4 & 11.2 & 3.12 \\
& -3 & 13.1 & 3.07 & -3 & 12.1 & 3.09 \\
& $-2(\mathrm{OLR})$ & 15.5 & 2.98 & -2 & 14.1 & 3.03 \\
& -1 & 23.0 & 2.58 & -1 & 20.0 & 2.75 \\
& $-1 / 2$ & 38.7 & 1.45 & $-1 / 2$ & 32.5 & 1.94 \\
\hline
\end{tabular}

where

$\kappa=\left[1-(c / a)^{2}\right]^{1 / 2}, \quad \lambda=\left[1-(b / a)^{2}\right] /\left[1-(c / a)^{2}\right]$,

$\mu=\lambda \sin ^{2} \theta \cos ^{2} \varphi$ and $v=-\left[\mu+\sin ^{2} \theta+\lambda \cos ^{2} \theta\right]$.

Similar expressions are found for the forces. The function $I(\theta, \varphi)$ and the corresponding functions for the forces were calculated numerically at intervals of $2^{\circ}$ in $\theta$ and $\varphi$, respectively, and the results were stored in tables. In the orbit calculations the angular dependence of the potential and force was evaluated by linear interpolation from these tables.

A system of units is defined by a unit of length $r_{0}=a=1$ and a unit of velocity $v_{0}=r_{0}\left(4 \pi G \varrho_{0}\right)^{1 / 2}=1$. The mass contained within a spheroid characterized by $m$ is :

$M=M_{0}\left(m / r_{0}\right)^{p+3}$ with $M_{0}=4 \pi a b c \varrho_{0} /(p+3)$.

Periodic orbits were calculated numerically with the method described by Magnenat (1982b). In a hamiltonian system an orbit is completely defined by its initial conditions $x_{0}, y_{0}, z_{0}, \dot{x}_{0}, \dot{y}_{0}, \dot{z}_{0}$. By fixing the energy and choosing a starting plane (e.g. $y_{0}=0$ ) four parameters $\left(x_{0}, z_{0}, \dot{x}_{0}, \dot{z}_{0}\right)$ are left, which are mapped on the same plane after a specific number of rotations. The 4 parameters are adjusted by a linear differential correction method until a periodic orbit is found. Linear stability is analysed using the linearized mapping from the point in the starting plane on itself.

With this method orbits in the plane perpendicular to the rotation-axis (the $z$-axis) were calculated at various energies. If an orbit became unstable to perturbations in the $z$-direction, an attempt was made to find a tilted orbit at the same energy.

The location of the resonances can be estimated by approximating the potential with spherical harmonics (derivations are omitted) :

$V(r, \theta, \varphi) \approx r^{p+2}\left[c_{00}+c_{20} P_{2}^{0}(\cos \theta)+c_{22} P_{2}^{2}(\cos \theta) \cos (2 \varphi)\right]$,

with the Associated Legendre Functions:

$P_{2}^{0}(x)=\frac{1}{2}\left(3 x^{2}-1\right) \quad$ and $\quad P_{2}^{2}(x)=3\left(1-x^{2}\right)$.

For $p=-1.8, c / a=0.5$ and $b / a=0.8$ :

$c_{00}=2.32094$,

$c_{20}=0.06486$,

and

$c_{22}=-0.00653$.
Neglecting the term with $\cos (2 \varphi)$ - thus retaining only the oblate part of the potential - and expanding around a fixed radius $r$ in the plane $z=0$ we obtain the frequencies:

$\omega_{0}=f_{0} r^{p / 2}$ with $f_{0}=\left[(p+2)\left(c_{00}-\frac{1}{2} c_{20}\right)\right]^{1 / 2}$,

$\omega_{1}=f_{1} \omega_{0}$ with $f_{1}=(p+4)^{1 / 2}$,

$\omega_{2}=f_{2} \omega_{0}$ with $f_{2}=\left[1+\frac{3 c_{20}}{\left(c_{00}-\frac{1}{2} c_{20}\right)(p+2)}\right]^{1 / 2}$.

Here $\omega$ is the rotation frequency of the triaxial system, $\omega_{0}$ the circular frequency in the plane $z=0, \omega_{1}$ the epicycle frequency in the same plane and $\omega_{2}$ the frequency in the $z$-direction. There is a horizontal resonance (in the plane $z=0$ ) when the ratio $\left(\omega_{0}-\omega\right): \omega_{1}$ can be represented by (small) integers. Vertical resonances occur when this is the case for the ratio $\left(\omega_{0}-\omega\right): \omega_{2}$. For brevity a notation $k: l: m$ is introduced to indicate the ratios $\left(\omega_{0}-\omega\right): \omega_{1}: \omega_{2}$. An asterisk is substituted if the frequency is not involved in the resonance. The locations of resonances are given by:

$r_{k: l: *}=\left[\frac{\omega}{f_{0}\left[1-f_{1}(k / l)\right]}\right]^{2 / p}$ for a horizontal resonance

and

$r_{k: *: m}=\left[\frac{\omega}{f_{0}\left[1-f_{2}(k / m)\right]}\right]^{2 / p}$ for a vertical resonance

With $p=-1.8, c / a=0.5$, and $b / a=0.8$ we have $f_{0}=0.677$, $f_{1}=1.483$, and $f_{2}=1.194$. Co-rotation (CR) is represented by $0: *: *$, the Inner Lindblad Resonance (ILR) by $1: 2: *$ and the Outer Lindblad Resonance (OLR) by $-1: 2: *$. Prograde orbits have $l / k>0$ within $\mathrm{CR}$ and $l / k<0$ outside CR. Equations (6) hold for retrograde orbits if $-\omega$ is substituted for $\omega$. The corresponding value of the energy $E=\frac{1}{2}\left(\dot{x}^{2}+\dot{y}^{2}+\dot{z}^{2}\right)-\frac{1}{2} \omega^{2}$ $\cdot\left(x^{2}+y^{2}\right)+V(x, y, z)$ is approximately:

$E=\frac{1}{2} r^{2}\left(\omega_{0}^{2}-2 \omega_{0} \omega\right)+\left(c_{00}-\frac{1}{2} c_{20}\right) r^{p+2}$.

Table 1 gives the approximate locations of the main resonances and the corresponding energies.

It should be noted that the term "energy" used here actually represents the Jacobi-integral in the co-rotating frame. For almost circular orbits, it increases with radius up to $\mathrm{CR}$ where it has its maximum, and then decreases as $r$ increases outside CR. Energy in its strict sense, as defined in the non-rotating frame with a time-dependent potential, is not a conserved quantity. However, far outside CR, the potential rotates relatively quickly with respect to an orbital period and can be looked upon as an effectively axisymmetric potential. The corresponding effective energy increases monotonically with radius.

\section{Resulting orbits}

There are 5 families of simple closed orbits in the plane perpendicular to the rotation axis: (1) the retrograde orbits, (2) the prograde orbits outside the OLR, (3) the prograde orbits inside the ILR, (4) the $x$-axial orbits, which are nearly linear at small radii, become more circular near the ILR and have complex shapes when passing through the higher order resonances up to $\mathrm{CR}$, and (5) the $y$-axial orbits, which exist only inside the ILR (see e.g. Contopoulos, 1980, 1981). At each vertical resonance there is a strip in the plane $z=0$ where the orbits are unstable to pertubations in the $z$-direction. At the edge 


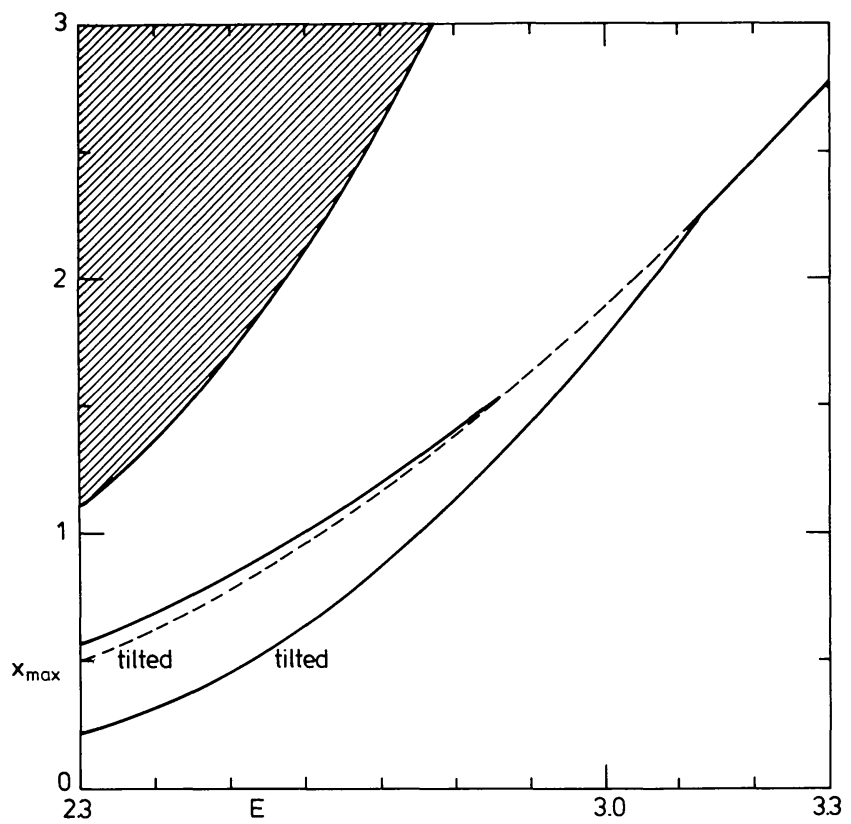

Fig. 1a. Bifurcation diagram of the $1: *: 1$ resonance, showing the retrograde tilted orbits branching off from the retrograde orbits in the plane $z=0$. Unstable orbits are indicated by dashes. In the shaded region no orbits can occur

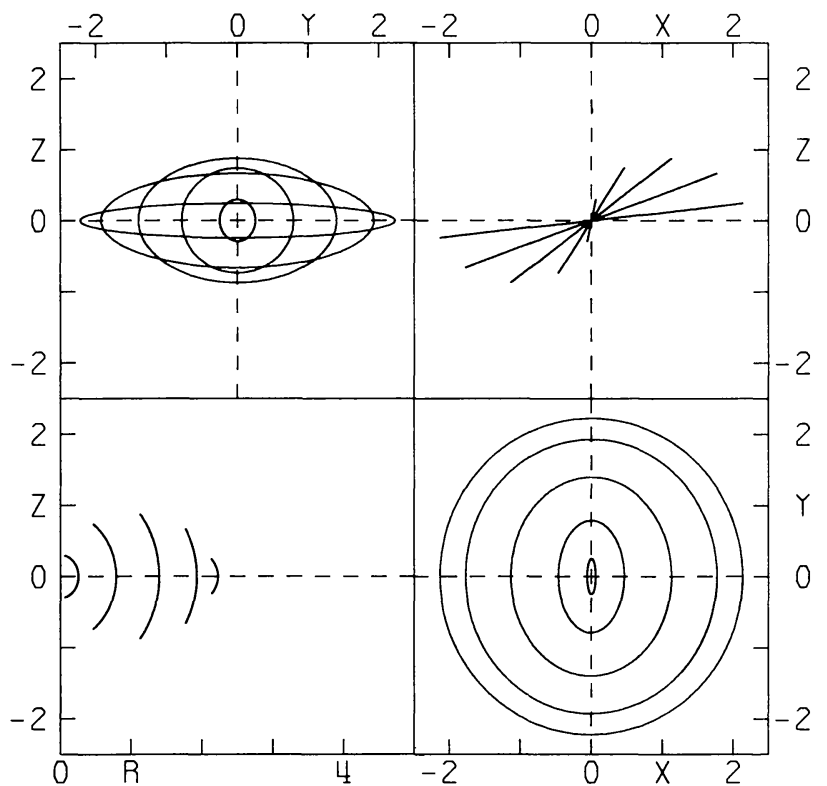

Fig. 2a. Stable retrograde orbits at the $1: *: 1$ resonance in various projections. The co-ordinate $R=\left(x^{2}+y^{2}\right)^{1 / 2}$. The plotted orbits have energies $E$ of $3.10,3.00,2.80,2.50$, and 2.00. The tilt with respect to the plane $z=0$ increases as $E$ decreases

of an instability strip a family of tilted orbits branches off from the orbits in the plane $z=0$.

Gas is likely to accumulate in simple stable periodic orbits. These were found at the $\pm 1: *: 1$ resonances, occurring within $\mathrm{CR}$ for the retrograde, and outside the OLR for the prograde orbits. The first type of orbits at the $1: *: 1$ resonance correspond to those described by Heisler et al. (1982). A more general analysis of this family was given by Magnenat (1982a).

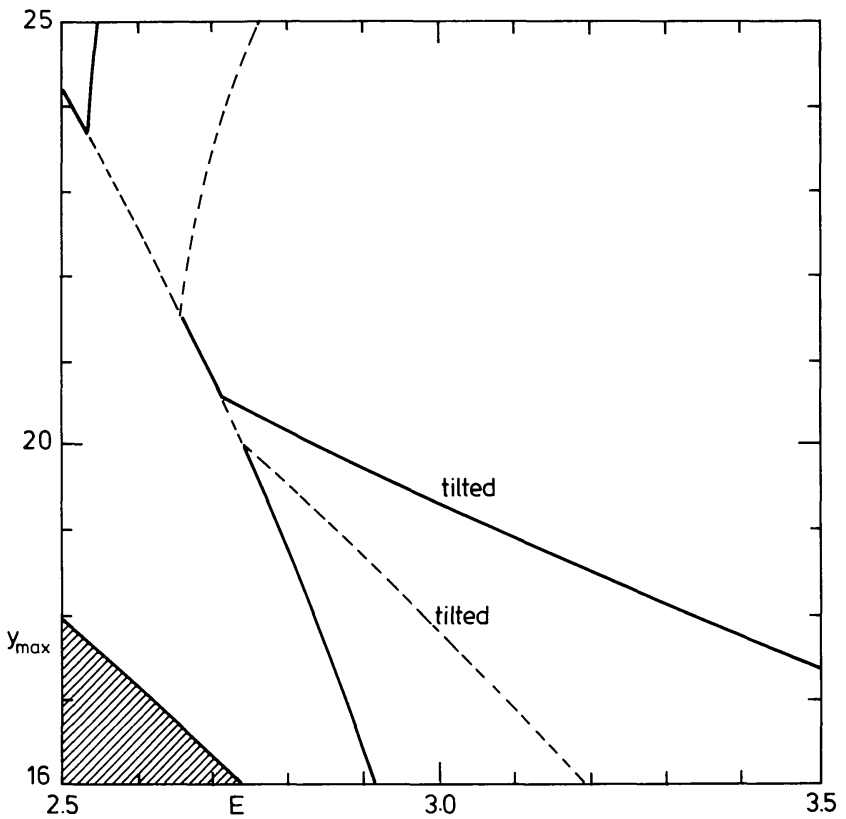

Fig. 1b. Bifurcation diagram of the $-1: *: 1$ resonance, showing the prograde tilted orbits. The $-1: 1: *$ resonance in the plane $z=0$ is shown as well

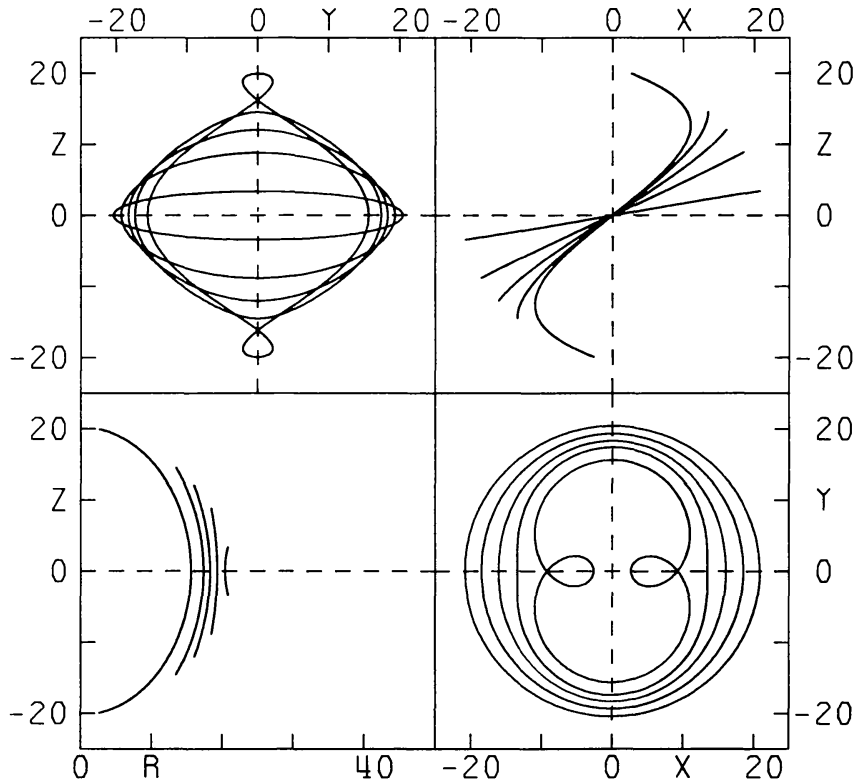

Fig. 2b. Stable prograde orbits at the $-1: *: 1$ resonance. The plotted orbits have energies $E$ of $2.75,3.00,3.25$, and 3.50. The orbits with the loops is at $E=4.40$. The tilt increases as $E$ increases

Figure 1a shows a bifurcation diagram of these orbits. One can make such a diagram by plotting two orbital parameters of orbits from several families. In this case the maximum $x$-value is plotted against the energy. In the shaded region, bounded by the zero velocity curve, no orbits can occur. Unstable orbits are indicated by dashed lines. The curve with the instability strip represents the orbits in the plane $z=0$. At the higher edge of the instability strip the stable tilted orbits branch off [at an energy 
somewhat larger than the approximate value predicted by Eq. (7) and given in Table 1]. The orbits are shown in Fig. 2a. Their distance with respect to the plane $z=0$ has a maximum in the direction of the major axis of the triaxial system (the $x$-axis). The tilt with respect to the plane $z=0$ increases as $E$ decreases. They become perpendicular to the $x$-axis at the origin, contrary to the work of Heisler et al. (1982) and Magnenat (1982a) where this occurs at a finite radius. This is due to the fact that the potentials used in those cases have cores.

At the lower edge of the instability strip at the $1: *: 1$ resonance in Fig. 1a an unstable family of tilted orbits branches off. The behaviour of these orbits is similar to that of the stable ones, but their maximum distance to the plane $z=0$ occurs in the direction of the $y$-axis.

A bifurcation diagram for the $-1: *: 1$ resonant orbits is given in Fig. 1b. The maximum $y$-value of an orbit is plotted as a function of energy. Again there is an instability strip with both a stable and unstable branch of tilted orbits. The stable ones are shown in Fig. 2b. They are tilted in the direction of the major axis of the triaxial system. The tilt increases as the Jacobiintegral $E$ increases. However, at higher values of $E$ they start to develop loops. The orbits with loops are still stable but gas cannot reside in them. This implies that gas in this type of orbit will have a maximum tilt.

The unstable branch of tilted orbits has the same behaviour as the stable one, but the maximum tilt is in the $y$-direction, just as is the case for the retrograde orbits.

The other instability strip shown in Fig. $1 \mathrm{~b}$ corresponds to the $-1: 1: *$ resonance in the plane $z=0$. There is a stable branch of orbits in the equatorial plane $z=0$ that look rather circular but have their centre shifted in the $y$-direction with respect to the origin. As these orbits intersect each other at varying energies they are not useful for explaining large scale asymmetries in the outer parts of galaxies (see e.g. Baldwin et al., 1980). The unstable branch consists of similar orbits shifted in the $x$-direction.

\section{Warps}

\subsection{General}

Gas of sufficiently low temperature is likely to settle down around the simple stable periodic orbits. If we assume this to be the case in the outer parts of a spiral galaxy, a connection can be made between the stable prograde tilted orbits at the $-1: *: 1$ resonance and a warp. In this way a warp can be explained as an almost stationary phenomenon.

There are two obvious characteristic properties of the described orbits that can serve as observational tests to check the correspondence between the orbits and warps. (1) The direction of the oval distortion or bar should coincide with the maximum tilt of the warp. (2) The location of the warp provides a length scale for the galaxy, predicting the location of the other resonances if the assumed density distribution is correct.

The location of the warp is mainly defined by the oblateness of the galaxy. From Eq. (6b) it follows that:

$r_{\text {warp }} / r_{\mathrm{CR}}=\left(1+f_{2}\right)^{-p / 2}$, where $f_{2}=\left(1+3 c_{20} / f_{0}^{2}\right)^{1 / 2}$.

Increasing the oblateness (by a more oblate halo or disk) enlarges $c_{20}$ and causes the location of the warp $r_{\text {warp }}$ to move farther outward with respect to CR. According to Binney (1981) the width of the instability strip increases as the strength of the oval distortion or bar increases. (Cutting of the bar at corotation would make the instability strip in Fig. 1b smaller.)

The question remains why the gas has settled in the tilted orbits and not in the plane of the galaxy. Binney $(1978,1981)$ proposed that the instability of orbits in the plane $z=0$ to perturbations perpendicular to that plane causes material to move out of the plane. The present results give strong support to this explanation and allow the development of a more complete scenario based on the assumption that gas remains close to the stable periodic orbits - thinking now in terms of clouds rather than a smooth distribution. Gas that is slowly spiraling inward around the stable prograde equatorial orbits will be forced to follow the stable tilted orbits when it reaches the instability strip, since these families are interconnected on the outside of the instability strip. The same dissipative process that makes the gas spiral inward - one may think of cloud-cloud collisions - causes it to move up to increasingly tilted orbits, until the point is reached where these orbits become self-intersecting. There, violent collisions will occur and the debris is likely to fall back to the equatorial plane. Such fragments may be observable as highvelocity clouds.

In the following sections, the results of Sect. 3 will be scaled to our Galaxy and used to make a rough model for NGC 2903.

\subsection{The warp of the Milky Way}

The assumed density distribution can be scaled to our Galaxy using the observed location of the warp and the velocity of the sun. In this way an estimate of the mass of the bulge can be obtained which should agree with the observational result if the prograde tilted orbits at the $-1: *: 1$ resonance correctly model a warp.

According to Kulkarni et al. (1982) the warp sets in at $18 \mathrm{kpc}$. In the model this occurs at a radius $r_{\text {warp }}=20.9$ (this value is derived from the orbit calculations and is slightly larger than the value in Table 1) implying a unit of length $r_{0}=0.861 \mathrm{kpc}$. Assuming the velocity of the sun to be $250 \mathrm{~km} \mathrm{~s}^{-1}$, as compared to a rotational velocity $v=\omega_{0} r=f_{0} r^{1+p / 2}=0.865$ at $10 \mathrm{kpc}$, yields the unit of velocity $v_{0}=289 \mathrm{~km} \mathrm{~s}^{-1}$. From these units it follows that $\varrho_{0}=\frac{1}{4 \pi G}\left(v_{0} / r_{0}\right)^{2}=1.4210^{-19} \mathrm{~kg} \mathrm{~m}^{-3}$ within $m=r_{0}$, resulting in a unit of mass $M_{0}=5.510^{9} M_{\odot}$. Thus, Eq. (3) can be written as: $M=6.610^{9}[\mathrm{~m}(\mathrm{kpc})]^{1.2} M_{\odot}$. This mass within a spheroidal volume characterized by $m$ is in reasonable agreement with Isaacman's (1981) result of $M=(9 \pm 2) 10^{9} M_{\odot}$ at $1 \mathrm{kpc}$, derived from the distribution of planetary nebulae near the galactic centre and consistent with the result of Sanders and Lowinger (1972) for the bulge of our galaxy.

From Henderson et al. (1982) the angle between the position of the sun and the major axis of the triaxial density distribution can be estimated to be $-80^{\circ}$. With Table 1 the locations of the main resonances can be predicted using the unit of length $r_{0}=0.861 \mathrm{kpc}$. There does not seem to be any relation between these locations and the observed structures in our Galaxy. Nor would this be expected, for the simple assumption of accumulating gas near the stable periodic orbits is certainly not satisfied within the solar radius.

\subsection{The warp of $\mathrm{NGC} 2903$}

The orbits plotted in Fig. $2 \mathrm{~b}$ can be projected on the sky to model NGC 2903. In this way the two main characteristic properties mentioned in Sect. 4.1 can be tested. 


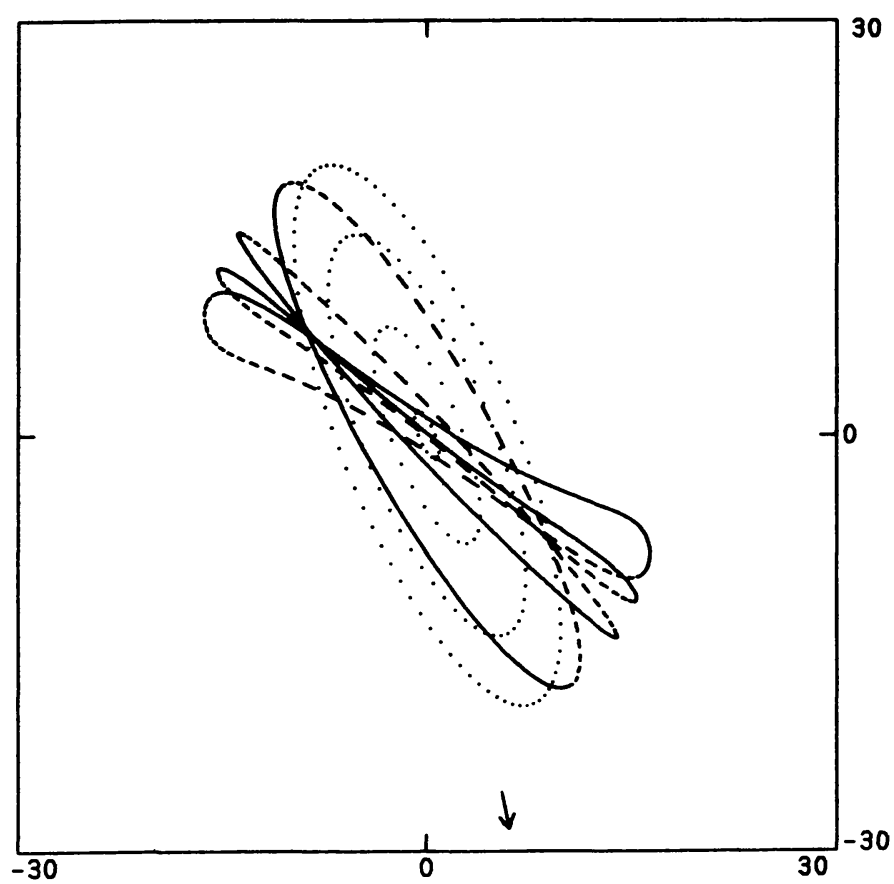

Fig. 3a. Projections on the sky of the prograde tilted orbits from Fig. $2 b$, the one with the loops excepted. The dotted lines are circles at consecutively the ILR, CR, OLR and the location of the warp. The arrow indicates the direction of the major axis of the bar or oval distortion

The relevant parameters for projection can be found from the results of optical spectral measurements by Simkim (1975) and neutral hydrogen measurements by Wevers (1983). Simkim (1975) found a position angle of the line of nodes PA $=28^{\circ} \pm 2^{\circ}$, an inclination $i=72.3 \pm 2^{\circ}$ and an angle between the major axis of the bar and the line of nodes of $43^{\circ}$ in the plane of the galaxy. The neutral hydrogen measurements by Wevers (1983) clearly show the warp of this galaxy. From the observed radial velocity field a position angle for the line of nodes $\mathrm{PA}=22^{\circ}$ can be derived. The position angle of the bar can be calculated from Simkim's results to be $12^{\circ}$. With a position angle $\mathrm{PA}=22^{\circ}$ for the line of nodes and an inclination $i=72.3$, it follows that the angle between the bar and the line of nodes is $30^{\circ}$. These parameters were used to project the orbits of Fig. 2b on the sky. Choosing the eastern side as the near side and letting the orbits come above the plane of the galaxy at the south west side yields Fig. 3a. The dotted lines indicate consecutively the ILR, CR, OLR and the location of the warp at model radii 1.86, 8.37, 15.5, and 20.9, respectively. The arrow indicates the direction of the oval distortion or bar. The orbits are dashed at the far side of the projection plane through the centre of the galaxy.

Figure $3 \mathrm{~b}$ shows the distribution of neutral hydrogen as observed by Wevers (1983). Comparing the model of Fig. 3a with the observations shows the following correspondences:

1. The central bar or oval distortion falls exactly within the co-rotation radius of the model when measured over its major axis. In the north, one of the two spiral arms starts where the major axis of the bar or oval distortion stops.

2. The hole in the neutral hydrogen distribution near the centre falls within the ILR.

3. The spiral arms extend to a radius between the OLR and the location of the warp. Observations of spiral galaxies general-

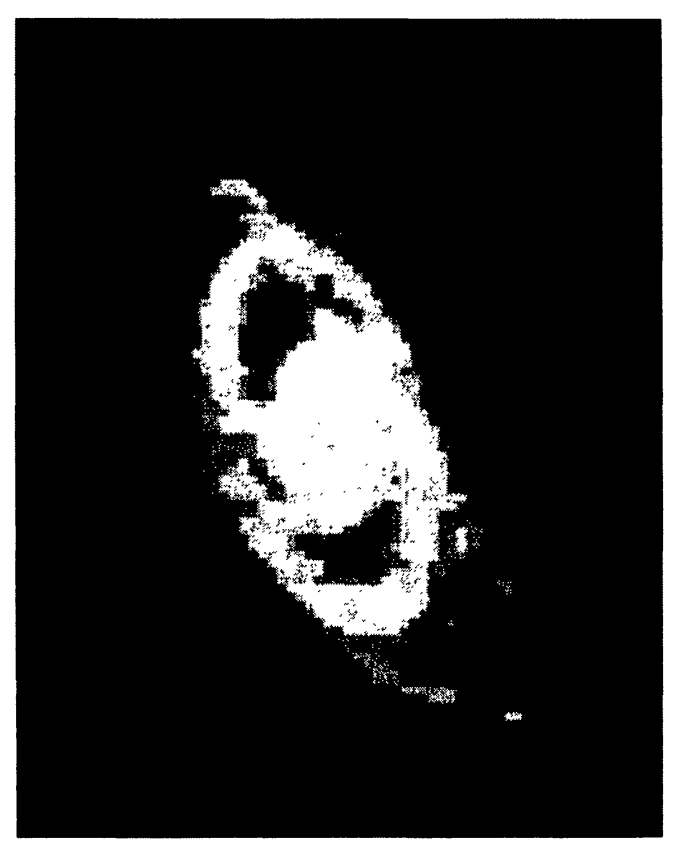

Fig. 3b. The neutral hydrogen distribution of NGC 2903 as measured by Wevers (1983). North is on top and east on the left. The horizontal length of the picture corresponds to $20^{\prime}$

ly show that the spiral arms become invisible at a radius somewhat beyond the OLR.

4. The point at which the orbits seem to intersect (they do not!), corresponding with the $y$-axis in the model, coincides in the south west with a bright spot. In the north east this is not clear.

5. When observing a distribution of gas in the projected orbits the turning points will show up most brightly. The turning points coincide with a strip of gas in the south west and less clearly on the opposite side.

These correspondences give good support to the model, at least qualitatively. The model predicts that in the southern part of the galaxy, anomalous velocities should be visible in highsensitivity neutral hydrogen measurements, and also in the north at places where the warp is not obscured.

\section{Conclusions}

The results presented above yield the following conclusions:

1. Under the assumption that the motion of gas in the outer part of a spiral galaxy can be described in terms of simple stable periodic orbits, a warp can be explained as an almost stationary phenomenon in a rotating triaxial system.

2. The interconnection between the stable prograde tilted orbits and those in the equatorial plane occurs on the outside of the instability strip. This gives strong support to the excitation of warps by orbital instability as proposed by Binney $(1978,1981)$. Gas spiraling slowly inward along the stable periodic orbits in the plane will be forced to continue along the tilted orbits and move progressively to the more inclined orbits of this family, until these become self-intersecting. Then violent collisions will 
occur. The debris that falls back to the equatorial plane may be observable as high-velocity clouds.

3. The location of the warp defines a length scale for the galaxy, related to the assumed density distribution. The maximum tilt in the warp defines the major axis of the bar or oval distortion. No severe contradictions are found when applying these results to the warp of our Galaxy and of NGC 2903.

Acknowledgements. I thank P. Magnenat for kindly supplying his program for orbit calculations, P.T. de Zeeuw for deriving the potential and for many stimulating discussions and B.M.H.R. Wevers for allowing me to copy Fig. $4 \mathrm{~b}$ prior to publication. I am indebted to Prof. W.W. Shane for giving the first impulse to this work. This paper has benefitted from comments and suggestions made by the referee, J.J. Binney, who also pointed out the astrophysical implications mentioned in Sect. 4.1.

\section{References}

Baldwin, J.E., Lynden-Bell; D., Sancisi, R.: 1980, Monthly Notices Roy. Astron. Soc. 193, 313

Becklin, E.E., Neugebauer, G.: 1968, Astrophys. J. 151, 145

Bertola, R., Galletta, G.: 1978, Astrophys. J. 226, L 115

Binney, J.J.: 1978, Monthly Notices Roy. Astron. Soc. 183, 779

Binney, J.J.: 1981, Monthly Notices Roy. Astron. Soc. 196, 455
Bosma, A.: 1978, The Distribution and Kinematics of Neutral Hydrogen in Spiral Galaxies of Various Morphological Types, Ph. D. Thesis, Univ. of Groningen, Chap. 5

Burstein, D., Rubin, V.C., Thonnard, N., Ford, W.K.: 1982, Astrophys. J. 253, 70

Chandrasekhar, S.: 1969, Ellipsoidal Figures of Equilibrium, Yale Univ. Press, New Haven

Contopoulos, G., Papayannopoulos, Th.: 1980, Astron. Astrophys. 92, 33

Contopoulos, G., : 1981, Astron. Astrophys. 102, 265

Hawarden, T.G., Elson, R.A.W., Longmore, A.J., Tritton, S.B., Corwin, H.G. : 1981, Monthly Notices Roy. Astron. Soc. 196, 747

Heisler, J., Merritt, D., Schwarzschild, M.: 1982, Astrophys. J. 258, 490

Henderson, A.P., Jackson, P.D., Kerr, F.J.: 1982, Astrophys. J. 263, 116

Isaacman, R.B.: 1981, Astron. Astrophys. 95, 46

Kulkarni, S.R., Blitz, L., Heiles, C. : 1982, Astrophys. J. 259, L 63

Liszt, H.S., Burton, W.B. : 1980, Astrophys. J. 236, 779

Magnenat, P.: 1982a, Astron. Astrophys. 108, 89

Magnenat, P.: 1982b, Celes. Mech. 28, 319

Sanders, R.H., Lowinger, T.: 1972, Astron. J. 77, 292

Simkim, S.M.: 1975, Astrophys. J. 195, 293

Van Albada, T.S., Kotanyi, C.G., Schwarzschild, M.: 1982, Monthly Notices Roy. Astron. Soc. 198, 303

Van der Kruit, P.C., Searle, L. : 1982, Astron. Astrophys. 110, 61

Wevers, B.M.H.R.: 1983, Ph. Thesis, Univ. of Groningen 